\title{
Challenges for Designing new Technology for Health and Wellbeing in a Complex Mental Healthcare Context
}

\author{
Anja Thieme ${ }^{1}$, John McCarthy ${ }^{2}$, Paula Johnson ${ }^{3}$, Stephanie Phillips ${ }^{3}$, Jayne Wallace ${ }^{4}$, \\ Siân Lindley ${ }^{1}$, Karim Ladha ${ }^{5}$, Daniel Jackson ${ }^{5}$, Diana Nowacka ${ }^{5}$, Ashur Rafiev ${ }^{5}$, Cassim Ladha ${ }^{5}$, \\ Thomas Nappey ${ }^{5}$, Mathew Kipling ${ }^{5}$, Peter Wright ${ }^{5}$, Thomas D. Meyer ${ }^{6}$ and Patrick Olivier \\ ${ }^{1} \mathrm{HXD}$, Microsoft \\ Research, UK \\ \{anthie@, \\ microsoft.com\} \\ ${ }^{2}$ School of Applied \\ Psychology, \\ University College \\ Cork, IRL \\ ${ }^{3}$ Calderstones \\ NHS \\ Foundation \\ Trust, \\ Whalley, UK \\ ${ }^{4}$ School of \\ Design, \\ Northumbria \\ University, \\ UK \\ ${ }^{5}$ Open Lab, \\ Newcastle \\ University, \\ UK \\ ${ }^{6}$ Department of \\ Psychiatry \& \\ Behavioral Science, \\ University of Texas, \\ US
}

\begin{abstract}
This paper describes the challenges and lessons learned in the experience-centered design (ECD) of the Spheres of Wellbeing, a technology to promote the mental health and wellbeing of a group of women, suffering from significant mental health problems and living in a medium secure hospital unit. First, we describe how our relationship with mental health professionals at the hospital and the aspirations for person-centric care that we shared with them enabled us, in the design of the Spheres, to innovate outside traditional healthcare procedures. We then provide insights into the challenges presented by the particular care culture and existing services and practices in the secure hospital unit that were revealed through our technology deployment. In discussing these challenges, our design enquiry opens up a space to make sense of experience living with complex mental health conditions in highly constrained contexts within which the deployment of the Spheres becomes an opportunity to think about wellbeing in similar contexts.
\end{abstract}

\section{Author Keywords}

Healthcare; fieldwork; hospital; experience-centered design; in-the-wild deployments; mental health; innovation.

\section{ACM Classification Keywords}

H.5.m. Information interfaces and presentation (e.g., HCI): Miscellaneous.

\section{INTRODUCTION}

In recent years, the topic of mental health has become a major concern to society due to increases in the occurrence of mental illness and the devastating effects it has on both the individual and the economy worldwide $[31,32]$. Surveys by

Copyright is held by the owner/author(s).

CHI 2016, May 07-12, 2016, San Jose, CA, USA

ACM 978-1-4503-3362-7/16/05.

http://dx.doi.org/10.1145/2858036.2858182

(c) (1)

This work is licensed under a Creative Commons Attribution International 4.0 License. the World Health Organization revealed that a large proportion of the people who suffer from mental disorders do not receive treatment $[2,61]$ due to the cost-intensive nature of many mental health therapies, social stigma preventing uptake, and adherence difficulties [18].

This has led the field of HCI to explore how to improve access to, and engagement with, therapeutic treatment. While research in this area is still young, there are important developments ranging from self-educational online therapies such as computerized Cognitive Behavioral Therapy (CBT) [19], to designs that supplement traditional psychotherapy programs. These include applications that assist the selfmonitoring of a person's thoughts, emotions or behaviors [i.e. 41, 49] including automatized assessments of a person's physical movements or social activity $[3,25]$; online social networks to help avert post-treatment relapse [34]; augmented and virtual reality systems to aid exposure treatment [63]; and game-like interfaces to support childtherapist communication $[12,46]$. The majority of these interventions are targeted at supporting therapeutic treatment, or the effective collection, visualization and analysis of patient data (cf. health informatics). Typically, they are informed in their understanding of mental health by the medical tradition that commonly defines it as absence of mental illness $[21,60]$.

In recent years, Corey Keyes [33], amongst others, has provided compelling evidence of empirical studies demonstrating that an absence of mental illness does not ensure the existence of positive mental health, and viceversa. Instead mental health and mental illness were found to present two distinct continua. He therefore argues for a more holistic view on mental health promotion that requires both the treatment of any illness (pathogenic focus) and the presence of wellbeing (salutogenic focus) - conceptualized as positive feelings towards, and positive functioning in life. Thus, effective mental health interventions should both support treatment and promotion of mental wellbeing [57]. Although the HCI community is beginning to respond to the general wellbeing agenda [e.g. 8, 13, 17, 29], research and designs for people suffering from severe mental disorders and who are hospitalized is extremely rare. 
This paper presents research insights from a $3 \frac{1}{2}$ year-long project, Spheres of Wellbeing, in which we designed and deployed technology for, and with, a group of women, who were sharing a six-bed flat in a medium secure unit (MSU) of a specialist National Health Service (NHS) hospital for people with a Learning Disability (LD). In addition to a mildto-moderate LD, the women had a diagnosis of Borderline Personality Disorder, a mental disorder characterized by severe emotion regulation deficits. Responding to their complex mental health and wellbeing needs and secure care environment, we developed the concept of the Spheres (see [55] for details) in collaboration with mental healthcare professionals (MHPs) at the hospital. The Spheres are a set of three artifacts that bring together qualities of physical objects and digital technology to invite engagements in therapeutic and mental wellbeing enhancing activities that feel personal and unique to the individual and help to reduce stigma and lower motivational barriers to engagement.

Against this backdrop of research in a relatively new area for $\mathrm{HCI}$, the paper makes two important contributions to the broader field of HCI research and design for mental health and wellbeing. Firstly, we describe and discuss how particular aspects of the good working relationships that we developed with MHPs at the hospital enabled us to innovate outside of traditional healthcare procedures and create new opportunities to design for personally meaningful experiences in this context. Secondly, we present an analysis of the deployment of the Spheres that surfaced key challenges for embedding technology within this complex mental health context that is governed by strict safety procedures and care guidelines. We highlight the principle drivers of this care culture and existing hospital practices that impact on technology adoption; and offer recommendations for identifying and addressing related challenges for others working in similarly complex or constrained care contexts.

\section{CONTEXT: WOMEN IN MEDIUM SECURE SERVICES}

Medium secure hospital units (MSU) provide treatment and care to people, who typically have come into conflict with the law and are detained under the Mental Health Act [43]. In this research, we collaborated with a NHS hospital that further specializes in the care and treatment of people with a Learning Disability. We focused especially on the women in this service, who typically suffer from significant mental health problems $(67 \%$ of female offenders with an Intellectual Disability in MSUs are diagnosed with a mental disorder [22]) and represent one of the most vulnerable patient groups. In addition to referrals by the criminal justice system, women also get admitted by community or mental health services [37] not because of any offending behavior, but due to the severity of their self-harming acts, or aggression towards other people or property that demand this higher level of secure care [22].

The majority of the women in the MSU have a diagnosis of Borderline Personality Disorder (BPD). BPD is a condition that is predominately associated with profound difficulties in regulating, modulating and tolerating emotions [64]. As sufferers of BPD, the women experience emotions more intensely and their mood tends to change rapidly between intense dysphoric emotions (i.e. extreme shame, panic or rage) to phases of euphoria, causing feelings of irritability, depression and anxiety [36]. In attempting to cope with intense negative emotions, the women tend to engage in behaviors that promise immediate relief or distraction, often displaying anger outbursts and violent behaviors; or engaging in self-harming behaviors such as cutting oneself, ingesting objects into the body, or self-neglect [35, 22]. Rooted in unpredictable emotional responses, they also exhibit a variety of cognitive disturbances that disrupt the development of their sense of self. For instance, they may hold strong beliefs that they do not deserve any kindness or feel guilty if they comfort themselves. All of these dysregulations impact upon their ability to have stable social relationships as well [45]. Their impulsive, often aggressive or severe self-harming behaviors, low mood and a general resistance to therapy mean that the women present a particularly vulnerable and challenging to treat group.

\section{Related Work}

The majority of existing mental health interventions are designed for outpatient services and, with few exceptions [i.e. 16], primarily for people with mild-to-moderate mental illness symptoms who are either attending or waiting for therapeutic treatment $[3,19,41]$, or engaging with online therapy and social network resources to help prevent relapse [34]. Important developments in this area include for instance Mind Balance [19], a guided online CBT program for people with depression. It is deliberately designed to be an engaging online experience, offering a wide range of, and flexible access to, diverse exercises for users to interact with; immediate feedback; as well as support by therapists and a social community of peers through the sharing of contents and personal stories. Findings from a clinical trial showed an increase in treatment adherence and an overall decrease in participant's depressive symptoms. In addition to demonstrations of their effectiveness in controlled settings [i.e. 2], both online and mobile-based approaches to treatment provide many advantages including ubiquitous access to therapy contents [28]; facilitating the recoding of patient data for self-assessment and reflection [3, 4] or therapist analysis [40]; and adaptability to a wide range of disorders [48]. Yet, they commonly face challenges for protecting collected patient data, lowering technology literacy barriers, or motivating patients to sustain engagements with therapy contents over time [19,34].

Furthermore, most interventions are based on written text, posing challenges to people with low literacy skills. Although people with LD are the population most at risk for developing mental health problems [35] with the prevalence of anxiety or mood disorders being twice that of the general population [47], they are often excluded from research. Issues of literacy have so far only been acknowledged in few existing designs for children [i.e. 12, 49]. For instance, in 
their self-monitoring phone application for children receiving fear therapy, Sa and Carrico [49] produced a series of drawings for children to manipulate to express the intensity of their fear (i.e. a weight lifting image to indicate the strength of perceived fear) rather than textual items. Initial findings of a clincial study revealed significant improvements in compliance with frequent fear charting, yet, more research and development is needed in this area.

HCI healthcare research that takes place in hospital settings commonly studies and describes challenges in technology adoption by clinicians [i.e. 5, 27] rather than the design of new technology for hospital inpatients [cf. 24]. Examples of technology deployments within MSU services are also very rare. Wallace et al. [59] for instance describe an interactive art piece for people with medium-to-severe symptoms of dementia within a mainly male adult MSU. While the authors report rich patient wellbeing experiences, challenges for technology adoption in this context are not discussed.

The Spheres research explores the unique challenges for designing and deploying a new therapy and wellbeing promoting technology for women with severe mental health problems, a LD, and who are living in a secure hospital.

\section{DESIGN PROCESS}

The HCI researchers' lack of clinical training or required qualifications for working in mental health or secure services together with the high vulnerability of the women due to the severity of their mental health problems meant that strict ethical considerations and procedures restricted any direct contact with the women patients in the early stages of this project. Even for less severe or constrained mental health contexts, the involvement of patients in the design stage is challenging, and thus, extremely rare (for exceptions see [3, 34]). Being restricted in the application of traditional usercentred and participatory design methods [11, 40], we followed a two-stage process that is commonly applied by HCI mental health researchers designing new interventions, whereby the technology is developed in close collaboration with MHPs at first, and then is clinically deployed [cf. 18].

Our collaboration with hospital staff involved the Research and Development (R\&D) manager of the hospital, a qualified nurse and CBT therapist with 15 years of experience working in the MSU. Due to her extensive clinical experience and personal concern for the women, the R\&D manager was keen to explore with us new ways to improve the situation of the women and became a champion for the project within the hospital. The 24-hour care of the women was organized and delivered by a large multi-disciplinary team of qualified nursing staff and unqualified support workers, who were assisted by psychiatrists, psychologists and allied health professionals such as Occupational Therapists (OT). Thus, in contrast with mental health designs for use by outpatients within their everyday life $[3,19,41]$ or in conjunction with a particular therapist or service [12, 46], our technology needed to fit with an integrated therapy model involving a multitude of staff and hospital services that operate within a hierarchically managed, safety-critical care setting; whose complexity poses a number of unique challenges to design.

To meet with MHPs at the hospital, the R\&D manager made on our behalf contact with, and selectively invited, members of the women's care team, who joined one or more of six informal meetings that were held at the hospital over a period of 12 months. Overall, these meetings were attended by a wide range of different mental health professionals including: 4 staff nurses working on the MSU; a ward and a clinical nurse manager; a consultant clinical psychologist; a safety manager; 3 research staff; and the medical director. In Thieme et al. [55], we detail how this collaboration with staff enabled us to gain important insights into the women's mental health needs, recommended treatments, and constraints of their secure care. To create a design that, as well as responding to staff's requirements, would respond to our vision of a personally meaningful mental health design, it was important for the staff to also understand common $\mathrm{HCI}$ research practices and opportunities offered by digital design. Next, we describe how we articulated our design vision in this regard to MHPs and how shared aspirations for person-centric care enabled us to innovate outside traditional healthcare procedures and informed the technology design.

\section{Innovating Outside Traditional Healthcare Procedures}

Many existing mental health interventions very closely follow the format and structure of traditional therapy programs. Likely, this is a consequence of the requirement to clearly demonstrate the potential benefits of a technology to patients without compromising their safety ("no harm to patients' rule) to ethics committees and MHPs in order to receive approval for a clinical technology deployment [cf. $18,24]$. This is most easily achieved where the technology builds on accepted treatment models; replicates, or is well integrated within already established clinical practices; and is used under guidance of MHPs. This however can limit the scope for design innovation in this area. Thus, rather than developing a technology 'solution' to fit with existing care practices [cf. 40], we intended (i) to enable new mental health and wellbeing supporting interactions that would feel personally meaningful to the individual; and (ii) to explore more openly how participants may use, adapt and integrate the technology into their everyday life on the MSU [cf. 9].

To describe the potential value and our vision for a more holistic, person-focused approach to the design and explain common HCI practices, we physically demonstrated, and showed to staff images and videos of, different technologies that were characteristic of our research approach [i.e. 56, 59]. For example, we introduced them to the concept of the Lovers' box [56], a technology that had the appearance of a wooden jewelry box and incorporated functionality that allowed romantic partners to exchange personal video messages. In this instance, we explained how in designing the artifact consideration was given to foregrounding a certain preciousness by using i.e. high quality materials and ornate decorations in the wood; and described how the 
interaction design emphasized a more symbolic relationship with the box related to the significant partner and qualities of intimacy and privacy rather than its technological features.

Examples such as this were used to explain to the staff our motivation for creating artifacts that enrich lived experiences, and that would be less perceived as a medical intervention that may unnecessarily foreground the women's psychosocial impairment or constraints of the secure setting. The designs chosen were often portable; utilized diverse interaction features such as RFID rather than i.e. keyboard or mouse inputs; and foregrounded a certain aesthetic and opportunities for personal experiences through the technology rather than its technical attributes or the device itself (e.g. a mobile phone). This challenged and expanded staff's often more conventional perceptions of IT as broadly meaning 'computers and screens', and invited curiosity about new possibilities for engaging the women that would sensitively respond to limitations in their cognitive abilities and feel stimulating, empowering and unique to them. Evolving conversations especially about strategies to achieve a personalized design received a lot of support by the staff; describing its fit with an ethos of person-centered care at the hospital, which seeks to put the needs of the women foremost and to actively involve them in decisions around their treatment to improve quality of care $[14,15,26]$. The shared ambition for a personal design and motivation to explore new, potentially more accessible technological interactions were key drivers in negotiating the design of the Spheres.

\section{Negotiating the Design of the Spheres}

In our meetings with MHPs, we introduced them to early sketches and prototypes of design ideas that were informed by our previous discussions; providing something concrete for them to imagine potential uses and offer critique. Our approach to engaging staff in dialogue about potential technology aligns well with previous research utilizing prototypes to assess i.e. usability issues and the general fit of a concept with existing services [62]; or to simulate their use in-situ to clarify potential adoption problems or training needs of therapists [42, 49]. Our collaboration helped address two key design challenges: (i) to identify, and effectively articulate to different MHPs, how the Spheres may benefit the women's therapy goals and present a fit with desirable hospital practices; and (ii) to achieve a design that would be considered as safe for women to use in the context of their challenging behavior and secure care environment.

Identifying \& Articulating the Potential Benefits of the Design Dialectical Behavioral Therapy (DBT) [e.g. 38, 44] is a specialist psychotherapy for the treatment of BPD that was in the process of being introduced to the women's care pathway in the low and medium secure services of the hospital. As such, especially higher level clinical managers proposed and strongly valued the idea of a technology that could build on the concept and practices of DBT to fit with the women's therapy goals. DBT usually involves group skills training sessions that teach the acquisition of skills that promote (i) behavioral change such as emotion regulation and inter-personal effectiveness; and (ii) acceptance including distress tolerance and mindfulness, teaching the person to accept the things they cannot change. The acceptance skills in particular account for the success of DBT and set it apart from other treatments for severe emotional regulation disorders [cf. 51]. To support distress tolerance, ward nurses and clinical therapists, described activities that offer visual or auditory stimulation (e.g. listening to music, imagining a safe place) or strong sensations (e.g. holding an ice cube in one's hands) as means of distraction instead of engagements in self-harm. For practices of mindfulness, the staff shared materials they use in therapy [39] and supplementary self-help resources by $[10,58]$ that are more accessible for LD populations.

Designed to complement practices of DBT acceptance skills and promote mental wellbeing, the Spheres are a set of three artefacts: the Mindfulness Sphere, Identity Sphere, and Calming Sphere (Fig. 1). The Mindfulness Sphere has the appearance of a crystal ball that, upon touch, assesses and reflects a person's heart rate through colorful lights that fadein and -out with every beat of their heart; thereby inviting focused attention to this visual stimuli to assist in practices of mindfulness. The Calming Sphere presents a non-digital bead-bracelet that the women can use for self-distraction when feeling slightly nervous or anxious; and the Identity Sphere plays back short videos that reflect personally meaningful contents related to the person to strengthen a positive self-image. For a detailed description of the design concept, rational and technical workings of the individual Spheres see $[54,55]$. To invite the women to also contribute to, and take ownership of their Spheres, we proposed that the principal researcher would assist each woman in creating a personalized look and contents for their artifacts through a series of making activities (i.e. create pieces to be enclosed inside of the Mindfulness Sphere, or videos for the Identity Sphere); and suggested that the Spheres be owned and used by the women at their own discretion, rather than through externally enforced or formally scheduled interactions.

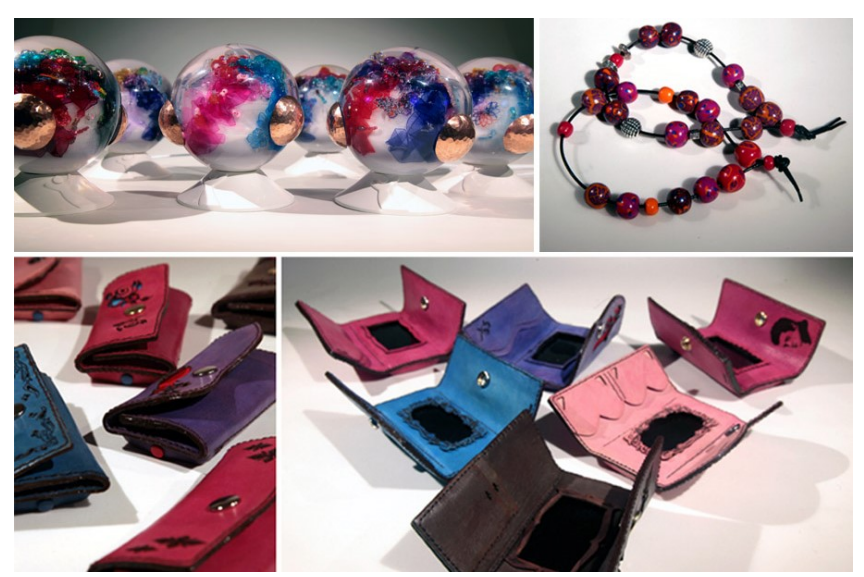

Figure 1. Spheres of Wellbeing: Mindfulness Spheres (top-left), Calming Spheres (top-right), and Identity Spheres (bottom). 
Discussing our design proposal, staff evaluated the Spheres' concept to resonate well with DBT and person-centered care. That the women would own the Spheres further meant that their adoption was not limited to uses in conjunction with Psychological Treatment Services (PTS), but provided scope for different ward staff and hospital services (i.e. OT) to take them on for person-centered activities with the women. This further aligns well with an integrated therapy model that the hospital was developing, whereby psycho-educational elements of treatment are delivered by the multi-disciplinary team of ward staff and therapy services.

\section{Aspirations for Safety vs. Portability \& Aesthetic Appeal}

Reviewing the concept of the three Spheres, hospital staff appreciated the physicality of the artifacts and liked that designed interactions were of little complexity (e.g. holding the artifact), yet highly visual, sensual and versatile; which they had recommended for people with LD, a disability that is characterized by deficits in attention span and literacy.

Much discussion however evolved around their portability. As a measure of safety, all furnishing on the MSU, including any tables, cupboards or beds were either safely attached to the floor or mounted against the wall. Free standing furniture was large and very heavy impeding it from being moved or picked up easily. While a situated installation on the MSU would likely reduce safety concerns regarding its robustness and in preventing access to any sharp pieces or batteries that could be used for self-harm, this contrasted with our design intent to create a more personalized technology. Considering that the women were under constant observation and often deprived of any personal possessions, we had favored for the Spheres to be portable to allow for interactions in private. More importantly, hospital staff valued the portability as it meant that the women could keep the artifacts when leaving the ward, which was a key concern by some, who assumed should the women truly enjoy using the technology - that a situated installation could interfere with their motivation to transition from the MSU to the lower secure services.

To identify suitable materials to create designs that were both portable and safe, staff showed us examples of curtains, blankets and soft padded mattresses that were specifically developed for secure and prison services; and whose fabrics and workmanship made them resistant to being ripped or damaged. To create safe objects that were also aesthetically satisfying however was a challenge that required trade-offs in design. For example, the Mindfulness Sphere is made from transparent resin allowing for it to encapsulate decorative pieces that remain visible to the eye. While resin was preferred to softer materials such as silicon that can be destroyed with one's teeth to get to the internal electronics, the more fragile nature and relative heavy weight of the Sphere however meant that risks remained related to it being potentially thrown at somebody, or broken into parts.

\section{DEPLOYMENT \& EVALUATION OF THE SPHERES}

Following approvals by the NHS Research Ethics Committee and our collaborating hospital for a trial of the
Spheres, we carefully recruited a group of six women, who shared a six-bedroom flat on the MSU. We refer to them as Sally, Kim, Janet, Lucy, Zoe and Alex (all pseudonym names). The majority of the women were in their twenties (age range: 18-43 years), and all had a mild-to-moderate LD (IQ range: 53-69) as well as a concurrent diagnosis of BPD, or a similarly severe emotion regulation disorder.

At first, their involvement included their participation in a series of five weekly sessions with the principal researcher during which they worked with different art and craft materials to creatively personalize the aesthetic appeal and contents of their set of Spheres (see [54] for detail on this process). Once the artifacts were finalized and handed to the women, this initiated a 15 week evaluation period. Initially, it was left open to the women how they might use the Spheres, so as to not restrict potential uses and to increase a sense of ownership and agency. At the end of week 1, the principal researcher then met individually with each woman to show uses of the Spheres that lend themselves to more therapeutic engagements. To capture the women experiences with the Spheres, we did interviews with them after the $1^{\text {st }}$ and $4^{\text {th }}$ deployment week that lasted on average 18 minutes $(\min =6, \max =25)$; did follow-up visits in week 10 and 15; and configured the two digital Spheres to collect interaction data (date, duration and frequency of use) over time.

In addition, we recruited 47 members of ward staff to support the research activities, which included supervisions of the creative sessions and interviews with the women, and recordings of their observations of the women's experiences of their Spheres on semi-structured event cards (postcards that can be completed quickly), and as more detailed diary entries. Using these recordings as prompts, we interviewed 17 of the staff about their impressions of the project and observations of the women at the end of deployment week four; these comprised of 8 qualified nurses (including one ward manager and a deputy), 7 day and 3 night support workers. Two interviews were conducted with pairs of staff and the remaining were one-to-one. The two pair interviews lasted for 38 and 45 minutes and individual interviews took on average 15 minutes $(\min =8, \max =22)$; governed by the time staff could spare while being on duty or their break. Individual staff are referred to using numbers (i.e. Staff-12).

All interviews were audio recorded, fully transcribed and subjected to Thematic Analysis (TA) [6]. TA is used to identify, analyze and report patterns within the data set; and to organize and describe it in rich detail. Frequently, it is used to also interpret various aspects of the research topic. Our TA included an intensive familiarization with the data, and the identification and systematic search for reoccurring themes in the data that were coded and developed into higher-level categories. In this paper we present our findings relating to challenges in the deployment that include the sub-themes: artifact safety, staff dynamics, ward culture and service integration; which evolved through this analytic process. 


\section{CHALLENGES FOR SPHERES APPROPRIATION IN-SITU}

This section begins with a brief overview of how the women appropriated their Spheres. Against this backdrop, we then present our findings of identified challenges in facilitating interactions with the Spheres within a complex, highly structured and socially dynamic hospital environment.

\section{Overview: Appropriation of the Spheres by the Women}

Throughout the project, the women expressed their enjoyment of the creative making sessions and their liking of the personalized Spheres, which they took great pride in. Log file data of the Mindfulness and Identity Spheres further showed how interactions with them were highest in the first two deployment weeks and had decreased over time with significant reductions in use beyond week four that marked the end of the core evaluation period during which the principal researcher had frequently visited and interviewed both the women and staff. For a detailed account of how the women made uses of the Spheres and how their experiences helped promote important facets of their mental health and wellbeing see [54]. Overall, we identified four main uses:

Firstly, the women frequently initiated acts of show-and-tell with members of the ward staff, hospital peers or their families during visits to explain the creation, significance and technical workings of the Spheres; providing opportunities for them to feel proud and receive recognition for their achievements and for positive social interactions. Secondly, they used the Spheres for self-reassurance by approaching them to be reminded of happy memories or good feelings; of their individuality; and of important people in their life; which felt empowering to some women, who described feeling more confident and motivated to try and progress in their life. Thirdly, they used the Spheres to keep well and maintain a sense of wellbeing when they felt lonely; were bored; sought stimulation or relaxation; or wanted to escape situations that would unsettle them. Here, uses of the Mindfulness Sphere especially were reported to have helped to relax and find calm, yet their use for practices of mindfulness was low and remained largely under-explored. Fourthly, the women were regularly prompted and directed by the ward staff to use their Spheres as tools for coping particularly when they were showing signs of distress - to distract them from their troubles. While the women mostly declined uses of the Spheres in those difficult moments, there were a multitude of reports of how engagements with the Spheres had helped to break cycles of disruptive thoughts.

Individual Risk Behaviors of Women: Concern for Safety The frequency and duration of Spheres' use was moderated by the extent to which the women had access to the artifacts, which was determined by the general safety of the designed objects and the individual risk behaviors of each woman.

Artifact Safety \& Staff's Readiness for Positive Risk Taking As with all materials or objects that were brought onto the MSU, staff were very cautious of potential safety risks involved in handing certain artefacts to the women. In terms of their safety, staff described the two digital Spheres as 'fairly robust' (Staff-07), 'low risk items' (Staff-43), particularly when compared to game consoles or CD players whose design does not restrict access to their internal electronics. While the Identity Spheres were considered by staff to be safe enough for the women to access when in distress and had frequently offered their use in those moments, concerns remained about the Mindfulness Sphere. As a 'fragile' and 'quite heavy' (Staff-40) object, most of the staff explained that they would not risk giving this Sphere to the women upon any signs of distress. Staff-29: “(...) I do know one staff, one or two staff have said, well, flippin' heck, if it's calming them down when they are anxious or angry, are we safe to be giving them, 'cause we've had televisions thrown at us and things, you know. You worry that, it's gonna get thrown back at you, if you give it them when they are angry, so we tend 'not' to offer it then."

The material configuration of the Spheres and the extent to which staff felt comfortable to take any risks by handing the Spheres to the women, was found to vary amongst them. Whereas some expressed aversion to taking any risks, others appeared more open to considering that 'it might be a risk worth taking' (Staff-07) and had a greater expectation of positive outcomes. For example, Staff-15 acknowledged: "(...) because the truth is, nobody has [had] anything thrown at them, nobody's smashed them, not to say it won't happen, but if somebody wants to throw one big heavy object, you know, it's plenty of big heavy objects in the rooms that they could throw anyway (...)."

This is somewhat reflective of the reality of everyday life on the ward, whereby the women were known to be able to identify different avenues for self-harm, if they had that intention. Adding her perspective about the risk behaviors of Kim, Staff-38 said: "It [pieces of the Spheres] wouldn't cause her any great harm 'cause she'd stuff it in her eye or whatever, it's not that she'd kill herself or anything with it."

\section{Spheres Access by the Women}

Assessments of the women's risk behaviors, such as a tendency to throw objects, determined if they could freely access their Spheres. Whilst the Spheres were typically stored behind a transparent Perspex cupboard together with other electronic appliances in the women's bedrooms, only two women had unrestricted access. All others had either temporary or no free access and needed to ask staff for their Spheres. Unsurprisingly, these restrictions were found to reduce opportunities for interactions. About Alex's frequent use of the Spheres Staff-22 i.e. explained: "Probably because she's got, she had access to it more than anybody else. Alex didn't have to involve anybody, did she, because she could just take it, herself off, if she were feeling low in mood, (...) where it's harder for the rest of them to access."

Requests by those women with restricted access for their artifacts from staff however were rarely reported. Some of the staff admitted that it may not come easy for the women, especially for Zoe who was often described as rather shy, to approach them. They also acknowledged that the women 
were aware that 'they can't access it as much' (Staff-22), unless individual staff support was available to facilitate.

\section{Need for Staff to Attend to Basic Care \& Hectic Ward}

For facilitating individual Spheres uses, staff frequently described being challenged by limitations in time and staff resources when attending to a hectic ward and in light of their primary concern of safeguarding the women and organizing their basic care (e.g. physical hygiene, medication intake, therapy attendance). Reflecting on why uses of the Spheres remained low after the initial weeks, Staff-29: “(...) maybe if we encouraged them more, but then again, it is basically at that time at night, we've got jobs on and, we try and rush everything, get everything done before the end of the shift and from that evenings tend to be the ones they have time to do it more, like I say, it's finding those times, keeping encouraging them."

Interactions with the Spheres also proved challenging if one or more of the women showed disruptive behaviors, or if an incident was occurring that demanded staff attention: "There just wasn't enough staff to facilitate, there was lots going on on the flat, staff were tied up, so they couldn't actually get that individual attention. We probably had to, level them from a certain area and we couldn't, sort of, split them up to do personal things, if that makes sense?" (Staff-47).

\section{Existing Work Practices \& Routines of the Staff}

Even at times when the ward environment was considered as settled, to facilitate uses of the Spheres was not always a straightforward process. For example, to unlock the Spheres cupboard, there was only one set of keys that was typically worn by one ward staff and shared between two separate hospital flats. To obtain these keys was at times problematic. In our conversations with Zoe in week 4, she described to not have been allowed to show her Identity Sphere to her mum and dad during a visit: "No, 'cause it's locked behind my thing [the Perspex cupboard]. There weren't enough staff on to take them out and stuff." When asked if Zoe had asked the ward staff to assist her, she asserted: "I did ask, they said next time maybe, when there are enough staff."

How even smallest undertakings could present a difficulty, particularly if they conflicted with already existing work routines, became further apparent in relation to the charging of the Spheres. To save energy, all electric appliances locked inside the Perspex cupboards were by default switched-off overnight. While a lack of power supply did not affect TV or stereo appliances, for the Spheres it could mean that they were not sufficiently charged to function when the women desired to use them; that is, until re-charged and re-set. Especially in the absence of the principal researcher, in weeks 5 to 15, the digital Spheres were often left un-charged; which was also captured in their data logs. Describing how any extra responsibilities that required staff's attention were at a risk of being perceived as an additional burden, Staff-22: “(...) to me it should have been their [the women's] responsibility, you know, the charging, you know, making it that it's been charged 24 whatever, you know, overnight, and that should have been their responsibility I think. But because a lot of them couldn't have access to it in their room you see, (...) a lot of the responsibility got put on to us."

\section{Staff's Difficulties in Attending to the Research Activities}

In addition to their propensity to facilitate engagements with the Spheres, staff members voiced difficulties in finding the time to document any observations they made of the women during or at the end of often busy, tiring and very long shifts (of up to 13 hours). They therefore described a preference for the easier and quicker to complete event cards above the diaries. Although staff expressed a desire to contribute to the research, the request for long, detailed accounts of their observations felt as 'overwhelming' (Staff-38) and 'hard work' (Staff-09). Staff also described their forgetting to fill in the diaries and difficulties to recall specifics of the events later in the day; and they described feelings of uncertainty in making sense of the women's experiences as well as discomfort about expressing their personal opinions about them, which contrasts to more factual presentations commonly required in the completion of clinical care notes. Staff-29 about the diary: "And lots of the questions were 'why do you think they...', well it's like reading their minds, we just, you just don't know 'cause you might be, what I think might not necessarily be right, it might be completely wrong."

\section{Existing Ward Culture vs. Understanding of DBT}

Further to pragmatic challenges in maintaining the Spheres technology and facilitating interactions, most ward staff were not very familiar with the care concept and practices of DBT. While the clinical care team of the women and higher-level ward or nursing managers, who were involved in the design of the Spheres, evaluated them to fit well with aspirations for person-centered care and DBT, DBT had only recently been introduced to the MSU. Thus, a large number of the ward staff were not yet trained about this therapy, which challenged their understanding of related practices such as mindfulness. Staff-05: "I suppose like, for example the DBT group is relatively new, just we are a little bit behind really, ideally we'd been doing it, using..., and I suppose aspects we've always done, so I think we are a bit behind in that (...) there is a bit of an absence in the staff training. I think the staff, it's not completely alien to them, but if you'd ask to sort of give a detailed account [about mindfulness] then the majority of people probably just remember what it is."

As mentioned earlier, the ward staff frequently directed the women to use their Spheres as tools for coping when they were showing signs of distress or going towards crisis; an approach that is common in behavior change interventions and one that the ward staff were familiar with. Although DBT teaches skills for promoting change, such as improved emotion regulation, the overall purpose of the program is to help the person create a sense of a life that they feel is worth living. Yet, throughout the deployment, we noticed how staffs' understanding of acceptance-based practices like mindfulness and how it could be cultivated was less well developed, restricting their ability to help guide the women 
in such like pursuits. Many of the staff therefore frequently asked for clear guidance as to how exactly we wanted them to instruct the women to use their Spheres, which conflicted with our more open-ended, exploratory research focus. Acknowledging limitations in her understanding of the Spheres for more therapy-focused uses, Staff-11: "I think, especially with the girls that we have got, would be mainly to show people, you know, and talk about the Spheres of Wellbeing, and you know, the creative, you know, the creative behind it, how it's made, as a therapeutic thing I don't, I don't know how it'd work (...).",

Since DBT and associated acceptance-based practices were not yet fully integrated into the ward culture, introducing the staff to new therapeutic concepts presents a complex, longerterm process that was likely to extend what could be achieved in individual briefings by the principal researcher and in the time scale of the project. To inform the ward staff about the research and how the Spheres related to DBT, the principal researcher provided written information in different formats (e.g. information booklets, email notifications, local A3 posters), made great efforts to meet with as many staff in person as possible (this is also reflected in the 47 staff who actively supported the project); and invited all staff to a showcase event where the women talked them through the details of the project. While all of this had helped to draw staff in, individual efforts by many of the staff in supporting the women with their Spheres often remained short-lived. Empathizing with this difficulty, Staff-43: "It could possibly to do with this environment though, hasn't it and, because it, it's quite a difficult environment to get someone from one-toone, careful or not, on the flats sort of using it and understanding it, and all staff being aware of, being able to [know] how to do it, how to advise them, how to use it properly, and charge it properly."

\section{Challenges for Service Integration}

Asking ward staff about how we could better support them and facilitate sustained Spheres uses, they often highlighted the need for a local technology champion and for integrating Spheres-related activities into existing hospital services.

\section{Need for Local Champion to Facilitate Engagements}

Unfortunately, for the deployment of the Spheres, one of the staff nurses, who had played a key role in the design process and championed the project on the MSU; was moved to a different hospital unit. Thus, in the absence of the principal researcher, there had not been a single person nominated to promote and continue activities around uses of the Sphere. Staff-11: "I don't know, it's almost like they've lost interest, you know, they don't have somebody there, who's specifically there for the Spheres of Wellbeing, or like yourself and [staff nurse], who were like heavily, you know, you were, are the [role] of Spheres of Wellbeing and so, I mean, you say 'C'mon let's have a look at your ball and things', they just, you know, it's difficult to get them to engage."

The women too approached us on many occasions, asking for continued engagement. When we asked them in week 15 how we could have supported them more, Alex suggested: "Persuade us!" and to get the staff to also persuade them; which Kim immediately expressed agreement with, asserting that the staff: "should offer you to, like, use them."

\section{Need for Integration into Existing Hospital Services}

In addition to a need for a more permanent technology champion on the ward, we identified difficulties as to how the research was introduced into the service. While the Spheres project arose out of our collaboration with hospital staff, it presented as an external research project that was not integrated with existing care practices. Exploring opportunities with staff as to how uses of the Spheres could become incorporated, many suggested that we embed for instance the Mindfulness Spheres into a regular group-based meditation or relaxation activity for the women, as this would formally set aside time for the ward staff to facilitate and supervise the engagement. Yet, group activities around the Spheres were complicated by the fact that one woman had moved to a lower secure unit one day prior to the deployment. This left both her - as the only woman in her flat with Spheres - as well as the newly admitted woman on the MSU, who did not possess any of the artifacts either, in an isolated position. This unequal distribution of Spheres on the wards prohibited any group activities. Consequently, staff often described the need to make the Spheres more 'widely available' (Staff-11) and to 'more patients' (Staff45); suggesting that the project "should be rolled out right across the NHS” (Staff-40) to facilitate sustained uses.

Similarly, while the clinical care team of the women was overall very supportive of the Spheres, an integration of their use into existing Psychological Treatment Services (PTS) proved challenging for two main reasons. Firstly, and as described above, DBT skills practice mostly takes place in groups, which were attended by women who did and did not possess Spheres. Secondly, while clinical psychologists could suggest and practice certain therapy-focused uses of the Spheres with the women during individual therapy sessions, these serve a very different purpose - that is to address personal or pressing problems such a self-harm or childhood trauma - and are therefore deemed too important to be substituted with an activity that presents a test of an, as of yet, un-proven technology and approach.

Moreover, service incorporation was further complicated by general difficulties to facilitate cross-service collaboration. Staff-05 for example described how the Spheres project potentially provided an opportunity for services offered by the Occupational Therapy (OT) team, who would be natural candidates to take on and continue the creative activities for the Spheres, and PTS to work closer together: “(...) perhaps there's a bit of a missing link between PTS and OTs, do you know what I mean, hopefully, you know if you are running an OT session with them, it should tie in with what they are doing in the therapies, and what they are trying to learn in anger management and bring that into art and all the rest of it. And perhaps that isn't as strong as it could be, so again, 
introducing these pieces of work, this project into those fields, OT and PTS, and getting them to work together a bit more maybe. (...) and again, I suppose it's getting that integrated approach isn't it really, which again is a really tough battle, but it needs to be changed and by everyone."

\section{DISCUSSION}

In the following, we discuss the challenges and practical insights from doing experience-centered design research in this rather complex social, professional and organizational context; and draw out lessons learned for other researchers working in similarly constrained health or care settings.

\section{Innovating Outside Traditional Healthcare Practices}

The value of building good working relationships with MHPs to identify requirements for, and iterate, design has frequently been recognized in HCI for mental health $[11,18]$. Building on this, we described explicit efforts to also explain our professional backgrounds and aspirations for an individualized, more holistic mental health design to MHPs. This was needed for hospital staff to be able to evaluate the potential benefits and risks of our less conventional technology proposal; which is a necessity for required project approvals. In this regard, we demonstrated how indepth explorations of examples of HCI designs that were characteristic of our personal-focused approach and sketches of the Spheres had been instrumental for stimulating dialogue and imagination about rich possibilities offered by digital design. This enabled important discussions about the potential value of exploring new interaction opportunities to sensitively respond to both the women's literacy skills and emotional needs. We further showed, how the identified shared ambition for a personal approach to supporting and empowering the women, that aligned well with hospital practices of person-centered care, as well as a clear positioning of the Spheres in relation to DBT- instead of a more general mental health and wellbeing agenda - became key drivers, alongside aspects of safety, in negotiations of the design. Thus, by achieving a better understanding of each other's professional practices and healthcare aspirations, we were able to identify a design space within which we could navigate and innovate more freely; and thereby avoid an over-reliance on traditional healthcare procedures.

\section{Identifying a Lead Research Champion}

Throughout this project, the R\&D manager played a key role in facilitating and governing the research on-site. It often was on the grounds of her personal commitment to the project and professional expertise that other clinicians' would make time to attend meetings and were open to support and build trust in the project. As a qualified nurse and CBT therapist, who had long-standing experience of working with the women, she had an intimate understanding of both patient needs as well as the daily running of the MSU. Her professional role further required the development of empathic working relationships with MHPs across the hospital; providing a network of connections to key personnel and members of the many different professions involved in the women's care. Holding a more senior work position and having a detailed understanding of hospital policies and management also meant that she would liaise, on our behalf, with higher level clinical managers to explain the purpose and relevance of the technology, which was instrumental to its acceptance. For working in such complex settings, this suggests identifying key study enablers that are not only enthusiastic about new opportunities for healthcare, but who also have an in-depth understanding of the target patients and the organization of their care; are well integrated within the large network of different staff professions involved; and have the expertise and competence to bring outsiders into this complex setting.

\section{Making Sense of Lived Experiences in Mental Health}

While strict ethical considerations and procedures restricted any contact with the women in the early design stage, working with MHPs as proxies had enabled rich insights into the women's mental health needs and care context [cf. 55]. Although, we were able to directly engage with the women as part of the deployment and ask them about their experiences with the Spheres, much of our understanding of them and their interactions continued to be meaningfully informed and contextualized by conversations with the staff. Partly, this was a consequence of observed struggles by the women to understand and articulate their own experiences, rooted in emotional difficulties related to their mental health condition. Furthermore, sudden declines in their mental health and frequent 'incidences' impacted on the ability of especially one woman to take part in scheduled research activities; which meant that we were more reliant on staff's observations especially during our absence from the hospital. While a need to remain flexible and responsive in research methods (i.e. when and how often to engage with the women) is generally recognized for both field deployments $[5,50]$ and working with mental health patients [23, 40], this was considerably amplified in this more extreme context.

As demonstrated in the findings, our analysis of the women's experiences considerably benefited from the personal observations and perspectives that the ward staff shared about the women. These were often meaningfully informed by their pre-existing (work-related) relationships with the women and included additional explanations of their work responsibilities in this setting. For doing ECD in challenging mental healthcare contexts, we therefore found continued consultations with staff to be essential for gaining important insights into the specifics of the care setting as well as a more holistic understanding of the experiences of people, who due to their mental health problems - may be more restricted in their ability to express themselves. To appropriately support staff in the sharing of their personal interpretations of the women, which was difficult for some as its contrasts with more factual clinical care notes, requires considerations for more support of staff, who are less experienced with qualitative research; and to design enquiry tools that are of little complexity and require minimal time to respond to, such as the event cards. Capturing snapshots of the women in-situ, these cards had been invaluable for prompting much richer accounts of staff's observations in later interviews. 


\section{Understanding the Principal Drivers of the Context}

Observations of how the women and staff engaged with the Spheres within their everyday life on the MSU deepened insight into the complexity of this context; and surfaced key challenges for technology adoption related to safety; primary work responsibilities of MHPs; and existing hospital culture.

\section{Requirement for Safety}

Despite our efforts to understand the physical configuration of the MSU and to appropriately respond to necessary safety requirements in the fabrication of the Spheres, risks remained particularly regarding the Mindfulness Sphere. In light of the women's behaviors of self-harm and aggression, this compromising of safety for achieving a design that was also portable, aesthetically appealing and offered compelling interactions meant that most women had only restricted access to the Spheres. This re-emphasizes the importance of safety in technology design for such contexts. Described safety perceptions of the Spheres by staff and how these informed their readiness to take 'positive risks' to facilitate engagements (anticipating beneficial outcomes), reflects the complexity involved in trying to simultaneously balance aspects of care with security; a challenge that extends to HCI research in similar contexts, such as design for advanced dementia [cf. 59], or probation units and prison services.

\section{Primary Care Responsibilities}

It is generally recognized in healthcare fieldwork [24, 40] that any activities requiring additional work of MHPs are difficult to sustain and can get compromised by other work responsibilities. For the MSU staff, making time to support Spheres interactions, however was especially complicated by some of the extremely challenging behaviors that the women would exhibit that can significantly disrupt any day-to-day activities and organization of their basic care. It is therefore important for HCI researchers to accept that, in contexts where staff's primary concern has to be the safeguarding of the patients, even the smallest undertakings (i.e. charging the Spheres) can present difficulties, and that opportunities for technology interactions are likely more limited.

\section{Existing Hospital Culture}

The deployment highlighted a discrepancy between existing work practices and desirable activities related especially to DBT, a therapeutic intervention newly introduced on the MSU. Thus, a large proportion of the ward staff had not yet been trained about DBT, which challenged their ability to support related activities such as mindfulness - with or without an inclusion of the Spheres. Instead, we observed more commonly how the ward staff would direct the women towards using the Spheres as 'tools for coping' when the women experienced distress. This reflects a more traditional care culture focused on treating problem behaviors rather than more acceptance-based, or preventative approaches. As the women often rely on staff support in their engagements with the technology, who play a key role in modelling behavior and assisting the women in their treatment goals, this highlights the importance of staff training and a shift in care culture; which is however complicated for specialized hospitals that are in constant flux and characterized by large volumes and high turn-arounds of staff (see also [52]).

\section{Opportunities for Innovation in Healthcare Practices}

In addition to insights into the specific circumstances within which engagements with the Spheres were situated, attempts to integrate their use into existing hospital services revealed additional challenges. These related to common issues of restricted resources [53] (i.e. limited availability of Spheres to more service users) as well as our approach to introducing the technology to participants and into the setting [7]. Designed specifically as personal possessions of the women, for use at their own discretion rather than embedding them i.e. as part of formal therapy sessions [cf. 40], as well as a lack of cross-service collaboration meant that only few staff would take initiative to facilitate engagements with the Spheres. While it was intended to stay open about how the women and staff may appropriate the Spheres, the need for staff to adhere to strict hospital procedures (to avoid injury and legal implications for the hospital), limited their ability to take risks in identifying own or alternative Spheres uses. Designed to coincide with innovations in approach, little consideration was given to develop specific practices around the Spheres that could be adopted by the ward staff and that were required to support new opportunities for mental health activities; which raises a broader question about the need to design practices around the technology. This suggest for future work to explicitly discuss strategies for new practices from the outset; and to work with patients and staff to translate successful examples of mental health and wellbeing activities identified in the deployment [cf. 54], into concrete practices for staff to adopt, and to assist the patients [cf. 20].

\section{CONCLUSION}

Research and design for people suffering from significant mental health problems and who are hospitalized is very rare. Designing and deploying the Spheres within an extremely challenging mental healthcare context enabled important insights into the complexity and specificity of the particular setting, and to explore new ideas for mental health and wellbeing technology in this space. We highlighted the value of our approach to working with MHPs for identifying new possibilities for a personal and accessible mental health design that avoided an over-reliance on traditional healthcare procedures. We provided practical insights and discussed our lessons learned about challenges for technology adoption that related to strict safety requirements; conflicting work responsibilities of staff; discrepancies with existing hospital culture; and a lack of service integration. These insights raised a broader question about the need to design new healthcare practices around the technology in future work.

\section{ACKNOWLEDGEMENTS}

We thank the six women and the hospital staff for their invaluable support of the research. This work was supported by Microsoft Research through its $\mathrm{PhD}$ Scholarship Programme and the RCUK Digital Economy Hub on Social Inclusion through the Digital Economy (SiDE). 


\section{REFERENCES}

1. Gerhard Andersson and Pim Cuijpers. 2009. Internetbased and other computerized psychological treatments for adult depression: A meta-analysis. Cognitive Behaviour Therapy 38(4), 196-205. http://dx.doi.org/10.1080/16506070903318960

2. Laura Helena Andrade, et al. 2014. Barriers to mental health treatment: results from the WHO World Mental Health surveys. Psychological Medicine 44(6), 1303-1317. http://dx.doi.org/10.1017/S0033291713001943

3. Jakob E. Bardram, Mads Frost, Károly Szántó, Maria Faurholt-Jepsen, Maj Vinberg, and Lars Vedel Kessing. 2013. Designing mobile health technology for bipolar disorder: a field trial of the monarca system. In Proceedings of the SIGCHI Conference on Human Factors in Computing Systems (CHI '13), 2627-2636. http://doi.acm.org/10.1145/2470654.2481364

4. Dror Ben-Zeev, Susan M. Kaiser, Christopher J. Brenner, Mark Begale, Jennifer Duffecy, David C. Mohr. 2013. Development and usability testing of FOCUS: A smartphone system for self-management of schizophrenia. Psychiatric Rehabilitation Journal 36, 289-296. http://dx.doi.org/10.1037/prj0000019

5. Ann Blandford, Erik Berndt, Ken Catchpole, Dominic Furniss, Astrid Mayer, Helena Mentis, Aisling Ann O'Kane, Tom Owen, Atish Rajkomar, and Rebecca Randell. 2014. Strategies for conducting situated studies of technology use in hospitals. Cognition, Technology \& Work, 1-14. http://dx.doi.org/10.1007/s10111-014-0318-7

6. Virginia Braun, and Victoria Clarke. 2006. Using thematic analysis in psychology. Qualitative Research in Psychology 3, 77-101. http://dx.doi.org/10.1191/1478088706qp063oa

7. Barry Brown, Stuart Reeves, and Scott Sherwood. 2011. Into the wild: challenges and opportunities for field trial methods. In Proceedings of the SIGCHI Conference on Human Factors in Computing Systems (CHI '11). ACM, 1657-1666. http://dx.doi.org/10.1145/1978942.1979185

8. Rafael A. Calvo, and Dorian Peters. 2013. Promoting Psychological Wellbeing: Loftier Goals for New Technologies. IEEE Technology and Society Magazine 32(4), 19-21.

http://dx.doi.org/10.1109/MTS.2013.2286429

9. Alan Chamberlain, Andy Crabtree, Tom Rodden, Matt Jones, and Yvonne Rogers. 2012. Research in the wild: understanding 'in the wild' approaches to design and development. In Proceedings of the Designing Interactive Systems Conference (DIS '12), 795-796. http://dx.doi.org/10.1145/2317956.2318078

10. Kimberly Christensen. 2009. Dialectical Behavior Therapy Skills, 101 Mindfulness Exercises and Other
Fun Activities for Children and Adolescents: A Learning Supplement. Bloomington: AuthorHouse.

11. David Coyle and Gavin Doherty. 2009. Clinical evaluations and collaborative design: Developing new technologies for mental healthcare interventions. In Proceedings of the SIGCHI Conference on Human Factors in Computing Systems (CHI '09), 2051-2060. http://doi.acm.org/10.1145/1518701.1519013

12. David Coyle, Nicola McGlade, Gavin Doherty, and Gary O'Reilly. 2011. Exploratory evaluations of a computer game supporting cognitive behavioural therapy for adolescents. In Proceedings of the SIGCHI Conference on Human Factors in Computing Systems (CHI '11), 2937-2946. http://doi.acm.org/10.1145/1978942.1979378

13. David Coyle, Anja Thieme, Conor Linehan, Madeline Balaam, Jayne Wallace, and Siân Lindley. 2014. Emotional wellbeing. International Journal of HumanComputer Studies 8(72), 627-628. http://dx.doi.org/10.1016/j.ijhcs.2014.05.008

14. Ara Darzi. 2008. High quality care for all: NHS next stage review final report. London: Department of Health.

15. Department of Health. 2011. Working with personality disordered offenders: A practitioners guide. Ministry of Justice, National Offender Management Service, London.

https://www.justice.gov.uk/downloads/offenders/menta lly-disordered-offenders/working-with-personalitydisordered-offenders.pdf

16. Colin A. Depp, Brent Mausbach, Eric Granholm, Veronica Cardenas, Dror Ben-Zeev, Thomas L. Patterson, Barry D Lebowitz and Dilip V. Jeste. 2010. Mobile interventions for severe mental illness: design and preliminary data from three approaches. The Journal of Nervous and Mental Disease 198(10), 715-721. http://dx.doi.org/10.1097/NMD.0b013e3181f49ea3

17. Pieter M. Desmet, and Anne E. Pohlmeyer. 2013. Positive design: An introduction to design for subjective well-being. International Journal of Design 7, 5-19. http://ijdesign.org/ojs/index.php/IJDesign/article/viewF ile/1676/586

18. Gavin Doherty, David Coyle, and Mark Matthews. 2010. Design and evaluation guidelines for mental health technologies. Interacting with Computers 22(4), 243-242.

http://doi.acm.org/10.1016/j.intcom.2010.02.006

19. Gavin Doherty, David Coyle, and John Sharry. 2012. Engagement with online mental health interventions: an exploratory clinical study of a treatment for depression. In Proceedings of the SIGCHI Conference on Human Factors in Computing Systems (CHI '12), 
1421-1430.

http://doi.acm.org/10.1145/2207676.2208602

20. Sara Donetto, Paola Pierri, Vicki Ysianakas, and Glenn Robert. 2015. Experience-based Co-design and Healthcare Improvement: Realizing Participatory Design in the Public Sector. The Design Journal 18(2), 227-248.

http://dx.doi.org/10.2752/175630615X1421249896431 2

21. Hugh Dubberly, Rajiv Mehta, Shelley Evenson, and Paul Pangaro. 2010. Reframing health to embrace design of our own well-being. interactions 17(3), 56-63. http://doi.acm.org/10.1145/1744161.1744175

22. Rebecca Fish. 2013. Women who use secure services: applying the literature to women with learning disabilities. The Journal of Forensic Practice 15(3), 192-205. http://dx.doi.org/10.1108/JFP-09-2012-0016

23. Mads Frost, and Steven Houben. 2014. Deploying healthcare technology 'in the wild': experiences from deploying a mobile health technology for bipolar disorder treatment. Fieldwork for Healthcare: Case Studies Investigating Human Factors in Computing Systems 2, 57-64.

http://dx.doi.org/10.2200/S00606ED1V02Y201410AR H007

24. Dominic Furniss, Rebecca Randell, Aisling Ann O'Kane, Svetlena Taneva, Helena Mentis, and Ann Blandford. 2014 (eds). Fieldwork for healthcare: guidance for investigating human factors in computing systems. Synthesis Lectures on Assistive, Rehabilitative, and Health-Preserving Technologies 3(2), 1-146.

http://dx.doi.org/10.2200/S00606ED1V02Y201410AR H007

25. Franz Gravenhorst, Amir Muaremi, Jakob Bardram, Agnes Grünerbl, Oscar Mayora, Gabriel Wurzer, Mads Frost, Venet Osmani, Bert Arnrich, Paul Lukowicz, and Gerhard Tröster. 2015. Mobile phones as medical devices in mental disorder treatment: an overview. Personal and Ubiquitous Computer 19(2), 335-353. http://dx.doi.org/10.1007/s00779-014-0829-5

26. Samantha Hall and Helen Duperouzel. 2011. "We know about our risks, so we should be asked." A tool to support service user involvement in the risk assessment process in forensic services for people with intellectual disabilities. Journal of Learning Disabilities and Offending Behaviour 2(3), 122-126. http://dx.doi.org/10.1108/20420921111186598

27. Thomas Riisgaard Hansen, Jakob E. Bardram, and Mads Soegaard. 2006. Moving out of the lab: Deploying pervasive technologies in a hospital. IEEE Pervasive Computing 5(3), 24-31. http://doi.ieeecomputersociety.org/10.1109/MPRV.200 6.53
28. Virginia Harrison, Judith Proudfoot, Pang Ping Wee, Gordon Parker, Dusan Hadzi Pavlovic and Vijaya Manicavasagar. 2011. Mobile mental health: Review of the emerging field and proof of concept study. Journal of Mental Health 20(6), 509-524.

http://dx.doi.org/10.3109/09638237.2011.608746

29. Marc Hassenzahl, Kai Eckoldt, Sarah Diefenbach, Matthias Laschke, Eva Len, and Joonhwan Kim. 2013. Designing moments of meaning and pleasure. Experience design and happiness. International Journal of Design 7(3), 21-31.

http://ijdesign.org/ojs/index.php/IJDesign/article/view/ 1480

30. Christopher J. James, John Crowe, Evan H. Magill, et al. 2009. Personalised Ambient Monitoring (PAM) of the mentally ill. In Proceedings of ECIFMBE 22, 1010-1013. http://dx.doi.org/10.1007/978-3-54089208-3 240

31. Ronald C. Kessler, et al. 2007. Lifetime prevalence and age-of-onset distributions of mental disorders in the World Health Organization's World Mental Health Survey Initiative. World Psychiatry 6(3), 168-176. http://www.ncbi.nlm.nih.gov/pmc/articles/PMC217458 $8 /$

32. Corey L. M. Keyes. 2010. The next steps in the promotion and protection of positive mental health. Canadian Journal of Nursing Research 42(3), 17-28.

33. Corey L. M. Keyes. 2014. Mental health as a complete state: How the salutogenic perspective completes the picture. In G.F. Bauer and O. Hämmig (eds.), Bridging Occupational, Organiztional and Public Health: A Transdisciplinary Approach. Springer, 179-192. http://dx.doi.org/10.1007/978-94-007-5640-3_1

34. Reeva Lederman, Greg Wadley, John Gleeson, Sarah Bendall, and Mario Álvarez-Jiménez. 2014. Moderated online social therapy: Designing and evaluating technology for mental health. ACM Transactions on Computer-Human Interaction 21(1), 1-26. http://doi.acm.org/10.1145/2513179

35. Marvin Lew, Cristy Matta, Carol Tripp-Tebo and Doug Watts. 2006. DBT for individuals with intellectual disabilities: A programme description. Mental Health Aspects of Developmental Disabilities 9, 1-12.

36. Klaus Lieb, Mary C. Zanarini, Christian Schmahl, Marsha M. Linehan and Martin Bohus. 2004. Borderline personality disorder. Lancet 364, 53-461. http://dx.doi.org/10.1016/S0140-6736(04)16770-6

37. William R. Lindsay, et al. 2004. Women with intellectual disability who have offended: Characteristics and outcome. Journal of Intellectual Disability Research 48(6), 580-590. http://dx.doi.org/10.1111/j.1365-2788.2004.00627.x 
38. Marsha M. Linehan. 1993. Cognitive-Behavioral Treatment of Borderline Personality Disorder. New York: The Guilford Press.

39. Marsha M. Linehan. 1993. Skills Training Manual for Treating Borderline Personality Disorder. New York: The Guilford Press.

40. Gabriela Marcu, Jakob E. Bardram, and Silvia Gabrielli. 2011. A framework for overcoming challenges in designing persuasive monitoring and feedback systems for mental illness. In IEEE Pervasive Computing Technologies for Healthcare (PervasiveHealth'11), 1-8. http://dx.doi.org/10.4108/icst.pervasivehealth.2011.246 097

41. Mark Matthews and Gavin Doherty. 2011. In the mood: engaging teenagers in psychotherapy using mobile phones. In Proceedings of the SIGCHI Conference on Human Factors in Computing Systems (CHI '11), 2947-2956. http://doi.acm.org/10.1145/1978942.1979379

42. Mark Matthews, Geri Gay, and Gavin Doherty. 2014. Taking part: role-play in the design of therapeutic systems. In Proceedings of the SIGCHI Conference on Human Factors in Computing Systems (CHI '14), 643-652. http://doi.acm.org/10.1145/2556288.2557103

43. Mental health care. 2013. Forensic mental health services.

http://www.mentalhealthcare.org.uk/forensic_mental_h ealth services

44. Catrin Morrissey, and Bridget Ingamells. 2011. Adapted dialectical behaviour therapy for male offenders with learning disabilities in a high secure environment: six years on. Journal of Learning Disabilities and Offending Behaviour 2(1), 8-15. http://dx.doi.org/10.5042/j1dob.2011.0024

45. Robert L. Palmer. 2002. Dialectic behaviour therapy for borderline personality disorder. Advances in Psychiatric Treatment 8(1), 10-16. http://dx.doi.org/10.1192/apt.8.1.10

46. Olga Pykhtina, Madeline Balaam, Gavin Wood, Sue Pattison, Ahmed Kharrufa, and Patrick Olivier. 2012. Magic land: the design and evaluation of an interactive tabletop supporting therapeutic play with children. In Proceedings of the Designing Interactive Systems Conference (DIS '12), 136-145. http://doi.acm.org/10.1145/2317956.2317978

47. Bronwyn Robertson. 2011. The adaption and application of mindfulness-based psychotherapeutic practices for individuals with intellectual disabilities. Advances in Mental Health and Intellectual Disabilities 5(5), 46-52. http://dx.doi.org/10.1108/20441281111180664
48. Marco de Sá, Luís Carriço and Pedro Antunes. 2007. Ubiquitous psychotherapy. IEEE Pervasive Computing 6(1), 20-27.

http://doi.ieeecomputersociety.org/10.1109/MPRV.200 7.23

49. Marco de Sá and Luís Carriço. 2012. Fear therapy for children: a mobile approach. In Proceedings of the 4th ACM SIGCHI symposium on Engineering interactive computing systems (EICS '12), 237-246. http://doi.acm.org/10.1145/2305484.2305524

50. Katie A. Siek, Gillian R. Hayes, Mark W. Newman and John C. Tang. 2014. Field deployments: Knowing from using in context. In J.S. Olson and W.A. Kellogg (eds.), Ways of Knowing in HCI, 119-142. Springer Science+Business. http://dx.doi.org/10.1007/978-14939-0378-8 6

51. Joaquim Soler, Juan Carlos Pascual, Thaïs Tiana, Anabel Cebrià, Judith Barrachina, M. Josefa Campins, Ignasi Gich, Enrique Alvarez, Víctor Pérez. 2009. Dialectical behaviour therapy skills training compared to standard group therapy in borderline personality disorder: A 3-month randomised controlled clinical trial. Behaviour Research and Therapy 47(5), 353-358. http://dx.doi.org/10.1016/j.brat.2009.01.013

52. Svetlena Taneva, Aisling Ann O'Kane, Raj Ratwani, Brian Hilligoss, Anja Thieme, and Kristina Groth 2014. Establishing and maintaining relationships in healthcare fields. Synthesis Lectures on Assistive, Rehabilitative, and Health-Preserving Technologies 3(2), 39-56.

http://dx.doi.org/10.2200/S00606ED1V02Y201410AR H007

53. Nick Taylor, Keith Cheverst, Peter Wright, and Patrick Olivier. 2013. Leaving the wild: lessons from community technology handovers. In Proceedings of the SIGCHI Conference on Human Factors in Computing Systems (CHI '13), 1549-1558. http://doi.acm.org/10.1145/2470654.2466206

54. Anja Thieme. 2015. Designing Technology to Promote Mental Health and Wellbeing. Doctoral Thesis, Newcastle University, UK.

http://designandwellbeing.com/papers/phd thesis Anja Thieme.pdf

55. Anja Thieme, Jayne Wallace, Paula Johnson, John McCarthy, Siân Lindley, Peter Wright, Patrick Olivier, and Thomas D. Meyer. 2013. Design to promote mindfulness practice and sense of self for vulnerable women in secure hospital services. In Proceedings of the SIGCHI Conference on Human Factors in Computing Systems (CHI '13), 2647-2656. http://doi.acm.org/10.1145/2470654.2481366

56. Anja Thieme, Jayne Wallace, James Thomas, Ko Le Chen, Nicole Krämer, and Patrick Olivier. 2011. Lovers' box: Designing for reflection within romantic 
relationships. Int. J. Hum.-Comput. Stud. 69, 5 (May 2011), 283-297.

http://dx.doi.org/10.1016/j.ijhcs.2010.12.006

57. Anja Thieme, Jayne Wallace, Thomas D. Meyer, and Patrick Olivier. 2015. Designing for mental wellbeing: towards a more holistic approach in the treatment and prevention of mental illness. In Proceedings of the 2015 British HCI Conference (British HCI '15), 1-10. http://doi.acm.org/10.1145/2783446.2783586

58. Carol Vivyan. 2009. An Introductory Self-Help Course in Cognitive Behaviour Therapy. Last retrieved $17^{\text {th }}$ Sept. 2015 from:

http://www.dbtselfhelp.com/SelfHelpCourse.pdf

59. Jayne Wallace, Anja Thieme, Gavin Wood, Guy Schofield, and Patrick Olivier. 2012. Enabling self, intimacy and a sense of home in dementia: an enquiry into design in a hospital setting. In Proceedings of the SIGCHI Conference on Human Factors in Computing Systems (CHI '12), 2629-2638.

http://doi.acm.org/10.1145/2207676.2208654

60. Alex M. Wood, and Nicholas Tarrier. 2010. Positive Clinical Psychology: A new vision and strategy for integrated research and practice. Clinical Psychology Review 30(7), 819-829.

http://dx.doi.org/10.1016/j.cpr.2010.06.003
61. World Health Organization. (2014). Mental Disorders: 2014 fact sheet. Last retrieved 6 ${ }^{\text {th }}$ Sept. 2015 from: http://www.who.int/mediacentre/factsheets/fs396/en/

62. Maja Wrzesien, Jean-Marie Burkhardt, Mariano Alcañiz Raya, and Cristina Botella. 2011. Mixing psychology and $\mathrm{HCI}$ in evaluation of augmented reality mental health technology. In CHI '11 Extended Abstracts on Human Factors in Computing Systems (CHI EA '11). ACM, 2119-2124. http://dx.doi.org/10.1145/1979742.1979898

63. Maja Wrzesien, Cristina Botella, Juana Bretón-López, Eva del Río González, Jean-Marie Burkhardt, Mariano Alcañiz, and María Ángeles Pérez-Ara. 2015. Treating small animal phobias using a projective-augmented reality system. Computers in Human Behavior 49(C), 343-353. http://dx.doi.org/10.1016/j.chb.2015.01.065

64. Shirley Yen, Caron Zlotnick and Ellen Costello. 2002. Affect regulation in women with borderline personality disorder traits. The Journal of Nervous and Mental Disease 100(10), 693-696. http://dx.doi.org/10.1097/01.NMD.0000034744.11140. 99 